\title{
ON THE NUMERICAL EVALUATION OF THE SINGULAR INTEGRALS OF SCATTERING THEORY
}

\author{
JAMES BREMER $^{*} \ddagger$ AND ZYDRUNAS GIMBUTAS ${ }^{\dagger}$
}

\begin{abstract}
In a previous work, the authors introduced a scheme for the numerical evaluation of the singular integrals which arise in the discretization of certain weakly singular integral operators of acoustic and electromagnetic scattering. That scheme is designed to achieve high-order algebraic convergence and high-accuracy when applied to operators given on smoothly parameterized surfaces. This paper generalizes the approach to a wider class of integral operators including many defined via the Cauchy principal value. Operators of this type frequently occur in the course of solving scattering problems involving boundary conditions on tangential derivatives. The resulting scheme achieves high-order algebraic convergence and approximately 12 digits of accuracy.
\end{abstract}

One of the principal observations of integral operator theory is that certain linear elliptic boundary value problems can be reformulated as systems of integral equations whose constituent operators act on spaces of square integrable functions [7, 20]. This observation plays a particularly important role in scattering theory, where such reformulations are standard $[14,11,15,13,12,7,8]$. Not surprisingly, it also figures prominently in the numerical treatment of scattering problems $[16,17,1,10]$. But while the integral equation approach to scattering theory is a venerable and well-developed subject, the corresponding numerical analysis - that is, the study of the integral equations of scattering theory using computers and finite precision arithmetic — is rather newer and considerably less developed. As a result, many fundamental problems in numerical scattering theory are as yet unresolved. Examples of this phenomenon can be found in recent contributions like [9] and [2], which offer new integral formulations of certain boundary value problems for Maxwell's equations that, unlike classical formulations, are amenable to numerical treatment.

This article concerns another unresolved problem: the evaluation of the singular integrals of scattering theory. A key difficulty in the discretization of those integral operators which arise from the reformulation of linear elliptic boundary value problems is the efficient and accurate evaluation of integrals of the form

$$
\lim _{\epsilon \rightarrow 0} \iint_{\Sigma \backslash B_{\epsilon}(x)} K(q, p) f(p) d s(p),
$$

where $\Sigma$ is a surface, $q$ is a point in $\Sigma, B_{\epsilon}(q)$ denotes the ball of radius $\epsilon$ centered at the point $q, K$ is singular kernel and $f$ a smooth function. The technique used to evaluate such integrals depends on the representation of the surface $\Sigma$ which is employed. Certain schemes are designed for triangulated surfaces [6], while others operate on the assumption that a smooth partition of unity given on $\Sigma$ is available [22]. The setting for this article is a parameterized piecewise smooth surface whose parameterization domain has been triangulated. More specifically, it is assumed that the surface $\Sigma$ is specified via a finite collection of smooth mappings

$$
\left\{\rho_{j}: \Delta^{1} \rightarrow \mathbb{R}^{3}\right\}_{j=1}^{N}
$$

given on the standard simplex

$$
\Delta^{1}=\left\{(s, t) \in \mathbb{R}^{2}: 0 \leq s \leq 1,0 \leq t \leq 1-s\right\}
$$

such that the sets

$$
\rho_{1}\left(\Delta^{1}\right), \rho_{2}\left(\Delta^{1}\right), \rho_{3}\left(\Delta^{1}\right), \ldots, \rho_{N}\left(\Delta^{1}\right)
$$

\footnotetext{
*Department of Mathematics, University of California, Davis, Davis CA 95616 USA.

${ }^{\dagger}$ Courant Institute of Mathematical Sciences, New York University, New York, NY 10012 USA.

${ }^{\ddagger}$ Corresponding author. E-mail address: bremer@math.ucdavis.edu.
} 
form a disjoint union of $\Sigma$. The quantities which must be evaluated in this case are of the form

$$
\lim _{\epsilon \rightarrow 0} \iint_{\Delta^{1} \backslash B_{\epsilon}(x)} K(\rho(x), \rho(y)) f(\rho(y))|d \rho(y)| d y,
$$

where $\rho: \Delta^{1} \rightarrow \mathbb{R}^{3}$ is a smooth mapping and $|d \rho(y)|$ denotes the determinant of the Jacobian of the mapping $\rho$ at the point $y$. Here, $x$ and $y$ refer to points in $\mathbb{R}^{2}$. It is our convention to use the symbols $p$ and $q$ to refer to points in $\mathbb{R}^{3}$ and $x$ and $y$ for points in $\mathbb{R}^{2}$. High accuracy, high-order techniques for the numerical evaluation of integrals of the form (2) are essentially nonexistent.

Indeed, standard techniques often perform poorly in the setting of parameterized surfaces even under the simplifying assumption that the kernel $K$ is integrable. Most existing methods proceed by applying a change of variables to the integral (2) in order to eliminate the singularity and then applying quadratures designed for smooth integrands. In the weakly singular case introducing polar coordinates centered at the target node $x$ results in an integral of the form

$$
\int_{0}^{2 \pi} \int_{0}^{R(\theta)} q_{-1}(\theta)+q_{0}(\theta) r+q_{1}(\theta) r^{2}+q_{2}(\theta) r^{3}+\cdots d r d \theta .
$$

Here, the coefficients $q_{j}(\theta)$ are periodic and analytic and the function $R(\theta)$ is periodic and piecewise analytic [18]. Applying tensor products of piecewise Gaussian quadratures in the variables $r$ and $\theta$ results in a sequence of approximations of (3) which converge exponentially — that is, with an error which behaves as

$$
O\left(\exp \left(-\lambda N^{1 / 2}\right)\right)
$$

in the number of quadrature nodes $N$. Unfortunately, while the functions $R(\theta)$ and $p_{j}(\theta)$ are analytic on the real line, they can have poles close to the real line. The poles of $R(\theta)$ depend on the location of the point $x$ vis-à-vis the boundary of the triangle $\Delta^{1}$ while those of the coefficients $q_{j}(\theta)$ are determined by the components of the metric tensor of the surface $\Sigma$ at the point $x$ in the basis of the tangent space induced by the parameterization $\rho$. Of course, when the poles of the $q_{j}(\theta)$ or of $R(\theta)$ are close to the real axis, which is the typical case in nontrivial scattering simulations, the constant $\lambda$ in (4) is small and convergence is retarded.

These difficulties with standard methods were the motivation for the article [4]. That work introduced a scheme for the high accuracy evaluation of the singular integrals arising from the discretization of integral operators which are linear combinations of the classical layer potential operators

$$
\begin{aligned}
S_{k} f(q) & =\iint_{\Sigma} G_{k}(q, p) f(p) d s(p), \\
D_{k} f(q) & =\iint_{\Sigma} \eta_{p} \cdot \nabla_{p} G_{k}(q, p) f(p) d s(p) \text { and } \\
D_{k}^{*} f(q) & =\iint_{\Sigma} \eta_{q} \cdot \nabla_{q} G_{k}(q, p) f(p) d s(p) .
\end{aligned}
$$

Here, $\eta_{p}$ denotes the outward-pointing unit normal vector to the surface $\Sigma$ at the point $p \in \Sigma, G_{k}(q, p)$ denotes the free space Green's function for the Helmholtz equation at wavenumber $k$

$$
G_{k}(q, p)=-\frac{1}{4 \pi} \frac{\exp (i k|q-p|)}{|q-p|} .
$$

That scheme obtains around 12 digits of precision, high-order algebraic convergence and, unlike standard mechanisms, its performance is largely insensitive to the behavior of the parameterization of the surface $\Sigma$. Indeed, the paper [4] describes several experiments in which the standard technique of applying tensor product Gaussian rules following a change of variables performed absurdly poorly due to small values of the constant $\lambda$ in (4). In one such experiment, Gaussian product rules with more than 100,000 nodes were required in order to match the accuracy of the quadrature rules of [4], which were of length less than 700.

This article extends the approach of [4] to operators which include terms of the form

$$
\begin{aligned}
& R_{k} f(q)=\lim _{\epsilon \rightarrow 0} \iint_{\Sigma \backslash B_{\epsilon}(x)} \nabla_{q} G_{k}(q, p) f(p) d s(p) \text { and } \\
& R_{k}^{*} f(q)=\lim _{\epsilon \rightarrow 0} \iint_{\Sigma \backslash B_{\epsilon}(x)} \nabla_{q} G_{k}(q, p) f(p) d s(p) .
\end{aligned}
$$


Operators of this type appear frequently in electromagnetic scattering computations, where they arise from problems involving boundary conditions on tangential derivatives. There are additional difficulties with such operators. After a change to polar coordinates, they lead to integrals of the form

$$
\lim _{\epsilon \rightarrow 0} \int_{0}^{2 \pi} \int_{\epsilon}^{R(\theta)} \frac{q_{-2}(\theta)}{r}+q_{-1}(\theta)+q_{0}(\theta) r+q_{1}(\theta) r^{2}+q_{2}(\theta) r^{3}+\cdots d r d \theta .
$$

The nonintegrable term makes the evaluation of (7) considerably more difficult that the evaluation of (3). A simple change of variable no longer suffices and standard techniques like those typically applied to weakly singular operators are no longer adequate.

Like the scheme of [4], the approach of this paper is limited to approximately 12 digits of precision. The loss of precision is due to round-off errors which occur in evaluating singular kernels such as the double layer potential

$$
\eta_{p} \cdot \nabla G_{0}(p, q)=\frac{(q-p) \cdot \eta_{p}}{|q-p|^{3}}
$$

naively. When $p \approx q$, cancellation errors in the numerator are magnified by division by a small quantity in the denominator. Higher accuracy can be obtained by expanding singular kernels in a Taylor series, but in order to obtain sufficient accuracy to make such an approach worthwhile, higher order derivatives of the surface parameterization must be available. We have chosen to evaluate kernels naively in the scheme of this paper because most integral equation solvers are expected to operate with only first order derivatives as inputs.

This article is structured as follows. In Section 1, we review a key tool of this article, generalized Gaussian quadrature. Section 2 gives a brief account of a Nyström method which captures the $L^{2}$ action of integral operators on surfaces. Section 3 concerns the construction of a set of precomputed quadrature formulae which enable the rapid evaluation of integrals of the form (2). Section 4 recounts numerical experiments which were conducted to test the efficacy of the scheme. We close with a few concluding remarks in Section 5.

\section{Generalized Gaussian quadrature}

This section concerns the numerical construction of quadrature rules of the form

$$
\int_{0}^{1} f(x) d x \approx \sum_{j=1}^{M} f\left(x_{j}\right) w_{j}
$$

which integrate a specified subspace $S$ of functions in $L^{2}([0,1])$. The subspace $S$ will be described by a spanning set $f_{1}, \ldots, f_{n}$ of functions which we will assume are pointwise defined in $(0,1)$. The condition that the quadrature rule (8) integrates functions in $S$ is equivalent to requiring that the system of equations

$$
\sum_{j=1}^{N} f_{i}\left(x_{j}\right) w_{j}=\int_{0}^{1} f_{i}(x) d x, \quad i=1, \ldots, n,
$$

be satisfied. Of course, since we concerned with a numerical procedure, we will have to content ourselves with quadrature rules for which (9) holds to a high degree of accuracy. We are interested in constructing quadrature rules of minimum possible length and we now describe a technique for obtaining quadrature formulae with approximately $\frac{1}{2} \operatorname{dim} S$ nodes. The discussion here is cursory; the article [5], which introduces the method, describes it in considerably more detail.

First suppose that $\left\{f_{1}, \ldots, f_{n}\right\}$ is an orthonormal set of functions in $L^{2}([-1,1])$ spanning the subspace $S$ and

$$
x_{1}, \ldots, x_{m}, w_{1}, \ldots, w_{m}
$$

are the nodes and weights of a quadrature which integrates products of the $f_{j}$, but with $m>>n$. Then a quadrature rule of length $n$ can be constructed by viewing (9) as a linear system with the weights $w_{j}$ as unknowns. More specifically, one forms the linear system of equations

$$
\left(\begin{array}{cccc}
f_{1}\left(x_{1}\right) \sqrt{w_{1}} & f_{1}\left(x_{2}\right) \sqrt{w_{2}} & \cdots & f_{1}\left(x_{m}\right) \sqrt{w_{m}} \\
f_{2}\left(x_{1}\right) \sqrt{w_{1}} & f_{2}\left(x_{2}\right) \sqrt{w_{2}} & \cdots & f_{2}\left(x_{m}\right) \sqrt{w_{m}} \\
\vdots & & \ddots & \vdots \\
f_{n}\left(x_{1}\right) \sqrt{w_{1}} & f_{n}\left(x_{2}\right) \sqrt{w_{2}} & \cdots & f_{n}\left(x_{m}\right) \sqrt{w_{m}}
\end{array}\right)\left(\begin{array}{c}
v_{1} \\
v_{2} \\
\vdots \\
v_{m}
\end{array}\right)=\left(\begin{array}{c}
b_{1} \\
b_{2} \\
\vdots \\
b_{n}
\end{array}\right)
$$


where

$$
b_{i}=\int_{0}^{1} f_{i}(x) d x, \quad i=1, \ldots, n .
$$

Because the rank of the matrix given the coefficients of this linear system is $n$, a rank-revealing QR factorization can be used to construct a solution

$$
\left(\begin{array}{c}
z_{1} \\
z_{2} \\
\vdots \\
z_{m}
\end{array}\right)
$$

of (10) with no more than $n$ nonzero entries. If we label those entries

$$
z_{i_{1}}, z_{i_{2}}, \ldots, z_{i_{n}}
$$

then

$$
\int_{0}^{1} f(x) d x \approx \sum_{l=1}^{n} f\left(x_{i_{l}}\right) z_{i_{l}} \sqrt{w_{i_{l}}}
$$

is a quadrature rule of length $n$ which integrates the $f_{j}$. Note that the matrix appearing in (10) has singular values which are either 1 or 0 owing to the assumption that the initial quadrature rule integrates products of the functions in the orthonormal set $\left\{f_{1}, \ldots, f_{n}\right\}$. The stability of this approach is discussed in more detail in $[5]$.

By viewing (9) as a nonlinear system of equations in the nodes $x_{j}$ and in the weights $w_{j}$, we can further reduce the length of the quadrature rule obtained by solving the linear system (10). Let

$$
y_{1}, \ldots, y_{l}, v_{1}, \ldots, v_{l}
$$

denote a quadrature formula which integrates the $f_{j}$. In order to reduce the length of the formula by 1 , we first delete one of the points in the quadrature rule and then use the remaining nodes and weights as an initial guess for the Gauss-Newton method, which is applied to the nonlinear system

$$
F_{i}\left(x_{1}, \ldots, x_{l-1}, w_{1}, \ldots, w_{l-1}\right)=b_{i}, \quad i=1, \ldots, n,
$$

where $F_{1}, \ldots, F_{n}$ are defined by

$$
F_{i}\left(x_{1}, \ldots, x_{l-1}, w_{1}, \ldots, w_{l-1}\right)=\sum_{r=1}^{l-1} f_{i}\left(x_{r}\right) w_{r}
$$

and the $b_{i}$ are as in (11). If suitable accuracy is obtained, then the reduced quadrature rule is accepted. If not, then another point is chosen and the quadrature rule obtained by deleting it is used as an initial guess for the Gauss-Newton method. This procedure is repeated until either a point is successfully eliminated or no point can be eliminated without reducing accuracy.

By repeatedly applying this algorithm for eliminating one quadrature node, a rule with approximately $\frac{1}{2} \operatorname{dim} S$ nodes can generally be formed.

Now suppose that the $f_{1}, \ldots, f_{n}$ specified by the user do not form an orthonormal basis for $S$, but are instead a spanning set with $n>\operatorname{dim} S$. First, an oversampled quadrature $x_{1}, \ldots, x_{m}, w_{1}, \ldots, w_{m}$ which integrates products of the given functions $f_{1}, \ldots, f_{n}$ is constructed via adaptive integration. Then an orthonormal basis for the span of $S$ is obtained by applying the pivoted Gram-Schmidt procedure to the columns of the matrix

$$
\left(\begin{array}{cccc}
f_{1}\left(x_{1}\right) \sqrt{w_{1}} & f_{2}\left(x_{1}\right) \sqrt{w_{1}} & \cdots & f_{n}\left(x_{1}\right) \sqrt{w_{1}} \\
f_{1}\left(x_{2}\right) \sqrt{w_{2}} & f_{2}\left(x_{2}\right) \sqrt{w_{2}} & \cdots & f_{n}\left(x_{2}\right) \sqrt{w_{2}} \\
\vdots & & \ddots & \vdots \\
f_{1}\left(x_{m}\right) \sqrt{w_{m}} & f_{2}\left(x_{m}\right) \sqrt{w_{m}} & \cdots & f_{n}\left(x_{m}\right) \sqrt{w_{m}}
\end{array}\right) .
$$

The resulting vectors give the scaled values of an orthonormal basis for the subspace $S$ at the nodes $x_{j}$. Note that the values of the orthonormal basis functions at any point in the interval $[0,1]$ can be computed via interpolation given their values at the nodes $x_{j}$. This basis is then used as an input to the procedure described above for computing a quadrature formula for functions in the subspace $S$. 


\section{AN $L^{2}$ NYSTRÖM METHOD}

We now give a brief account of a Nyström method for the discretization of integral operators of scattering theory on smooth surfaces. This approach differs from standard Nyström methods in that it captures the $L^{2}$ action of an operator rather than its pointwise behavior. A more detailed account of the approach described here can be found in [4] and a discussion of the advantages of $L^{2}$ discretization over standard Nyström and collocation methods can be found in [3].

2.1. Decompositions and discretization quadratures. By a decomposition of a surface $\Sigma \subset \mathbb{R}^{3}$ we mean a finite sequence

$$
\left\{\rho_{j}: \Delta^{1} \rightarrow \Sigma\right\}_{j=1}^{m}
$$

of smooth mappings given on the simplex $\Delta^{1}$ with non-vanishing Jacobian determinants such that the sets

$$
\rho_{1}\left(\Delta^{1}\right), \rho_{2}\left(\Delta^{1}\right), \ldots, \rho_{m}\left(\Delta^{1}\right)
$$

form a disjoint union of $\Sigma$.

We call a quadrature rule $\left\{x_{1}, \ldots, x_{l}, w_{1}, \ldots, w_{l}\right\}$ on $\Delta^{1}$ with positive weights which integrates all elements of the space $\mathscr{P}_{2 N}$ of polynomials of degree at most $2 N$ on the simplex $\Delta^{1}$ a discretization quadrature of order $N$. Associated with a discretization quadrature is the mapping $\Lambda: \mathscr{P}_{N} \rightarrow \mathbb{C}^{l}$ given by

$$
\Lambda(p)=\left(\begin{array}{c}
p\left(x_{1}\right) \sqrt{w_{1}} \\
p\left(x_{2}\right) \sqrt{w_{2}} \\
\vdots \\
p\left(x_{l}\right) \sqrt{w_{l}}
\end{array}\right)
$$

which embeds $\mathscr{P}_{N}$ isomorphically into $\mathbb{C}^{l}$. In the event that the rule $x_{1}, \ldots, x_{l}, w_{1}, \ldots, w_{l}$ is Gaussian - that is, its length $l$ is equal to the dimension $(N+1)(N+2) / 2$ of the space $\mathscr{P}_{N}$ - the mapping $\Lambda$ is invertible. For the most part, Gaussian quadratures on triangles are not available. In the experiments of this paper, we use generalized Gaussian quadrature rules which integrate polynomials of degree less than or equal to $2 N$ but which had more than $(N+1)(N+2) / 2$ nodes. Table 1 lists the properties of the discretization quadrature rules used in this paper.

\begin{tabular}{cccc} 
Discretization order $(N)$ & Integration order & Length & Dimension of $\mathscr{P}_{N}$ \\
\hline 4 & 8 & 17 & 15 \\
8 & 16 & 52 & 45 \\
12 & 24 & 112 & 91 \\
16 & 32 & 192 & 153
\end{tabular}

TABLE 1. The properties of the discretization quadrature rules used in this paper.

2.2. Discretizations of the integral operators. We now associate with a decomposition

$$
D=\left\{\rho_{j}: \Delta^{1} \rightarrow \Sigma\right\}_{j=1}^{m}
$$

and discretization quadrature $\left\{x_{1}, \ldots, x_{l}, w_{1}, \ldots, w_{l}\right\}$ a scheme for the discretization of certain integral operators. We begin by letting $S$ be the subspace of $L^{2}(\Sigma)$ consisting of all functions $f$ which are pointwise defined on $\Sigma$ and such that for each $j=1, \ldots, m$ the function

$$
f\left(\rho_{j}(x)\right)\left|d \rho_{j}(x)^{*} d \rho_{j}(x)\right|^{1 / 2}
$$

is a polynomial of order $N$ on $\Delta^{1}$. Denote by $P$ be the projection of $L^{2}(\Sigma)$ onto the subspace $S$ and let $\Phi: S \rightarrow \mathbb{C}^{m l}$ be a mapping which takes $f \in S$ to a vector with entries

$$
f\left(\rho_{j}\left(x_{i}\right)\right) \sqrt{w_{i}}\left|d \rho_{j}\left(\rho_{j}\left(x_{i}\right)\right)^{*} d \rho_{j}\left(\rho_{j}\left(x_{i}\right)\right)\right|^{1 / 2}, \quad j=1, \ldots, m, \quad i=1, \ldots, l .
$$

The ordering of the entries of $\Phi(f)$ is irrelevant. If $H: L^{2}(\Sigma) \rightarrow L^{2}(\Sigma)$ is of the form

$$
H f(x)=\lim _{\epsilon \rightarrow 0} \iint_{\Sigma \backslash B_{\epsilon}(x)} K(x, y) f(y) d s(y),
$$


with $K$ a linear combination of the kernels listed in (5) and (6), then we call the $m l \times m l$ matrix $A$ such that the diagram

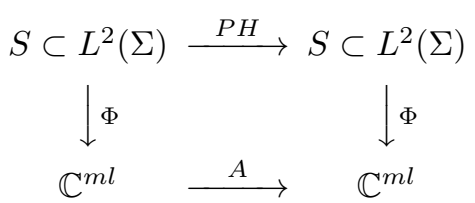

commutes the discretization of the operator $H$ induced by the specified decomposition and discretization quadrature. One of the advantages of this $L^{2}$ Nyström method is that the convergence theory mirrors the standard Galerkin convergence theory for integral equations. Indeed, the matrix $A$ is related to the Galerkin discretization of $H$ on the subspace $S$ via composition with matrices whose condition numbers are the condition number of the discretization quadrature $\left\{x_{1}, \ldots, x_{l}, w_{1}, \ldots, w_{l}\right\}$.

2.3. A convergence result. We now exploit the relationship between this Nyström method and Galerkin methods to establish a convergence result. Let $\left\{D_{j}\right\}_{j=1}^{\infty}$ be a sequence of decompositions of a surface $\Sigma$ and fix an order $N$ discretization quadrature $Q=\left\{x_{1}, \ldots, x_{l}, w_{1}, \ldots, w_{l}\right\}$. Designate by $S_{j}$ the subspace of functions associated with $D_{j}$ and $Q$ and let $P_{j}: L^{2}(\Sigma) \rightarrow S_{j}$ be the orthogonal projection $L^{2}(\Sigma) \rightarrow S_{j}$. Moreover, for each $j$, we let $A_{j}$ be the discretization of an integral operator $H$ of the form (12) corresponding to $D_{j}$ and $Q$.

We will assume that the sequence $\left\{P_{j}\right\}$ of projections is consistent and stable; that is, $P_{j} f \rightarrow f$ for all $f \in C^{\infty}(\Sigma) \subset L^{2}(\Sigma)$ and $\sup _{j}\left\|P_{j}\right\|_{2}<\infty$. Under these conditions, if we form a sequence $\left\{f_{j}\right\}$ of approximations of the solution $f$ of the integral equation

$$
(I+H) f=g
$$

by solving the sequence of linear systems

$$
\left(I+A_{j}\right) f_{j}=P_{j} g,
$$

then it follows from the standard theory for Galerkin methods (a discussion of which can found, for instance, in [10]) that

$$
\left\|f-f_{j}\right\|_{2}=O\left(\left\|f-P_{j} f\right\|_{2}\right) .
$$

If the right-hand side $g$ is smooth, the solution $f$ of (14) will be as well, and hence can be represented by piecewise polynomials. In this case, we expect our approximations to achieve $N$ th order algebraic convergence.

This assumes, however, that the entries of the matrices $A_{j}$ are computed correctly. Doing so requires a scheme for the evaluation of integrals of the form

$$
\lim _{\epsilon \rightarrow 0} \iint_{\Delta^{1} \backslash B_{\epsilon}(x)} K(x, \rho(y)) f(\rho(y))|d \rho(y)| d y .
$$

When the target node $x$ is sufficiently distant from the surface $\rho\left(\Delta^{1}\right)$, the integral (15) can be evaluated using the discretization quadrature. But, there are two difficult cases in which such an approach does not suffice: when the target node $x$ lies on the surface $\rho\left(\Delta^{1}\right)$ (the singular case) and when the target node is close to, but not inside, the surface $\rho\left(\Delta^{1}\right)$ (the nearly singular case). This article addresses the first, singular case. In the experiments of this paper, the nearly singular case was treated via adaptive quadrature in the manner described in Section 2.3 of [4].

\section{QUADRATURES FOR THE SINGULAR INTEGRALS OF SCATTERING THEORY}

In this section, we describe an approach to the evaluation of integrals of the form

$$
\lim _{\epsilon \rightarrow 0} \iint_{\Delta^{1} \backslash B_{\epsilon}(x)} K(\rho(x), \rho(y)) f(\rho(y))|d \rho(y)| d y,
$$

where $\rho: \Delta^{1} \rightarrow \mathbb{R}^{3}$ is a smooth map given on the standard simplex $\Delta^{1}, d \rho(y)$ denotes the Jacobian of the mapping $\rho$ at the point $y,|d \rho(y)|$ is the determinant of that Jacobian, $f$ is a smooth function and $K$ is a linear combination of the kernels listed in (5) and (6).

Our goal is to construct quadrature formulas which are accurate to a given algebraic order $N$. This ensures that the scheme does not interfere with the asymptotic rate of convergence of the discretization method described in Section 2. 
3.1. An adaptive change of variables. Most schemes for the evaluation of integrals arising from elliptic boundary value problems proceed by first changing to polar coordinates centered at the target point $x$. In the case of the integral (16), this results in an expression of the form

$$
\lim _{\epsilon \rightarrow 0} \int_{0}^{2 \pi} \int_{\epsilon}^{R(\theta)} \frac{q_{-2}(\theta)}{r^{2}}+\frac{q_{-1}(\theta)}{r}+q_{0}(\theta)+q_{1}(\theta) r+q_{2}(\theta) r^{2}+\cdots r d r d \theta,
$$

where $R(\theta)$ is the distance from the origin of the intersection of the ray of angle $\theta$ through $x$ and the boundary of $\Delta^{1}$. Here, the coefficients $q_{j}(\theta)$ are periodic and analytic on the real line and the function $R(\theta)$ is piecewise analytic [18]. Unfortunately, by itself this change of variables does not put the integrand of (16) into a tractable form. The coefficients $q_{j}(\theta)$ and the function $R(\theta)$ can have poles arbitrarily close to the real line. The efficiency of standard quadrature techniques like generalized Gaussian quadrature and the trapezoid rule depends on the domain of analyticity of the integrands and they converge slowly in the presence of poles close to the real axis.

The proximity to the real line of the poles of the $q_{j}(\theta)$ is a measure of the extent of non-conformality of the mapping $\rho$ at the target node $x$. Let $\left\{\xi_{1}, \xi_{2}\right\}$ denote the basis of the space tangent to $\Sigma$ at the point $x$ induced by the parameterization $\rho$; that is,

$$
\xi_{1}=\frac{\partial \rho\left(x_{1}, x_{2}\right)}{\partial x_{1}} \quad \text { and } \quad \xi_{2}=\frac{\partial \rho\left(x_{1}, x_{2}\right)}{\partial x_{2}} .
$$

Then, as shown in [19], the poles $w$ of the coefficient $q_{j}(\theta)$ are given by the equation

$$
\cot (w)=-\lambda^{-1} e^{ \pm i \gamma}
$$

where

$$
\cos (\gamma)=\frac{\xi_{1} \cdot \xi_{2}}{\left|\xi_{1}\right|\left|\xi_{2}\right|} \quad \text { and } \quad \lambda=\frac{\left|\xi_{1}\right|}{\left|\xi_{2}\right|} .
$$

Note that when $\lambda=1$ and $\cos (\gamma)=0$ - that is, when the mapping $\rho$ is conformal at the point $x$ - the $q_{j}(\theta)$ are entire. Similarly, the location of the poles of the function $R(\theta)$ depend on the proximity of the target node $x$ to the boundary of the triangle $\Delta^{1}$.

In order to simplify the integrand of (16), our approach begins by applying an affine change of variables which is adapted to the parameterization $\rho$. We introduce a triangle $T$ and an affine mapping $A: T \rightarrow \Delta^{1}$ such that the composed mapping $\tilde{\rho}=\rho \circ A: T \rightarrow \mathbb{R}^{3}$ is conformal at the point $x_{0}=A^{-1}(x)$. The mapping $A$ and triangle $T$ can be obtained by computing a singular value decomposition

$$
d \rho(x)=U \Sigma V^{*}
$$

of the Jacobian of $\rho$ at the point $x$, letting

$$
A=V \Sigma^{-1}
$$

and setting $T=A^{-1}\left(\Delta^{1}\right)$. As a consequence of the conformality of the mapping $\tilde{\rho}$ at the point $x_{0}$, when

$$
\lim _{\epsilon \rightarrow 0} \iint_{T \backslash B_{\epsilon}\left(x_{0}\right)} K(\tilde{\rho}(x), \tilde{\rho}(y)) f(\tilde{\rho}(y))|d \tilde{\rho}(y)| d y,
$$

is rewritten in the form

$$
\lim _{\epsilon \rightarrow 0} \int_{0}^{2 \pi} \int_{\epsilon}^{\tilde{R}(\theta)} \frac{\tilde{q}_{-2}(\theta)}{r}+\tilde{q}_{-1}(\theta)+\tilde{q}_{0}(\theta) r+\tilde{q}_{1}(\theta) r^{2}+\tilde{q}_{2}(\theta) r^{3}+\cdots d r d \theta
$$

by introducing polar coordinates centered at the point $x_{0}$, the coefficients $\tilde{q}_{j}(\theta)$ are entire. In fact, more can be said: not only are the $\tilde{q}_{j}(\theta)$ entire in this case, they are actually trigonometric polynomials of finite order. Indeed, $\tilde{q}_{-2}(\theta)$ is of the form

$$
\alpha \cos (\theta)+\beta \sin (\theta)
$$

while for $j>-2$, the order of $\tilde{q}_{j}(\theta)$ is bounded above by $3(j+1)+2$. This can be shown using a trivial modification of the arguments of [18].

This adaptive change of variables is helpful, but by itself insufficient. It does nothing to address the fact that the function $\tilde{R}(\theta)$ can have poles close to the real axis. Indeed, in many cases, the behavior of the parameterization $\tilde{R}(\theta)$ of the triangle $T=A^{-1}\left(\Delta^{1}\right)$ is considerable worse than that of the function $R(\theta)$ in this respect. 
3.2. Decomposition of the integration domain. Our approach now calls for the domain of integration $T$ to be split into two pieces - a circle $B_{\gamma}\left(x_{0}\right)$ of radius $\gamma>0$ centered at $x_{0}$ and its complement $T \backslash B_{\gamma}\left(x_{0}\right)$. That is, the integral (18) is decomposed as

$$
\begin{gathered}
\lim _{\epsilon \rightarrow 0} \iint_{B_{\gamma}\left(x_{0}\right) \backslash B_{\epsilon}\left(x_{0}\right)} K(\tilde{\rho}(x), \tilde{\rho}(y)) f(\tilde{\rho}(y))|d \tilde{\rho}(y)| d y+ \\
\iint_{T \backslash B_{\gamma}\left(x_{0}\right)} K(\tilde{\rho}(x), \tilde{\rho}(y)) f(\tilde{\rho}(y))|d \tilde{\rho}(y)| d y .
\end{gathered}
$$

The constant $\gamma$ must be positive and $B_{\gamma}\left(x_{0}\right) \subset T$, but it is otherwise arbitrary. For the experiments of this paper, we let $\gamma$ be $1 / 2 d$, where $d$ is the distance from the point $x_{0}$ to the boundary of the triangle $T$. Figure 1 depicts this decomposition.
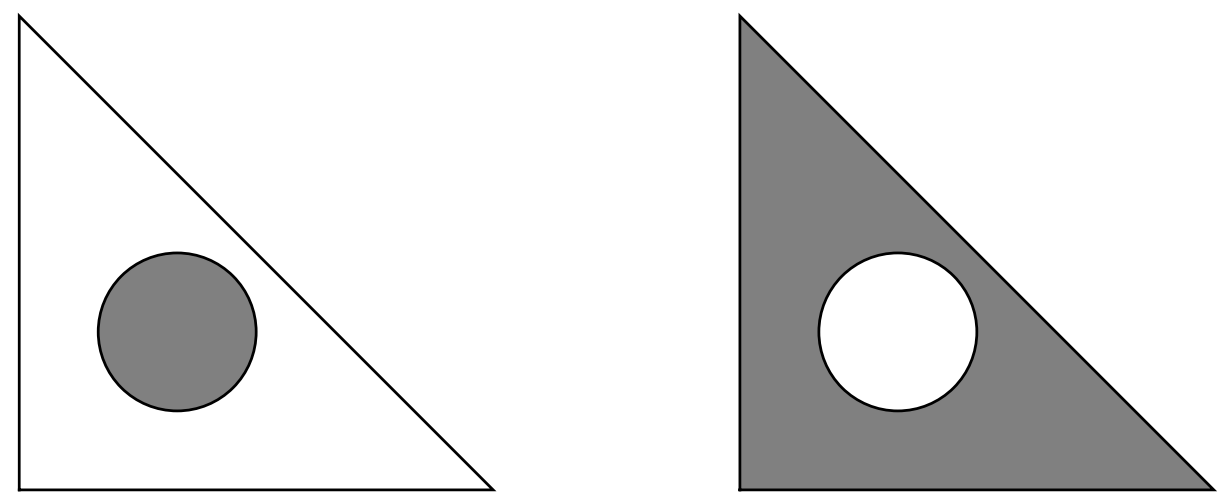

Figure 1. The domain of the integral (18) is first divided into the two shaded regions shown here.

The first term in (20) can be evaluated to $N$ th order algebraic accuracy by rewriting the integral as

$$
\lim _{\epsilon \rightarrow 0} \int_{0}^{2 \pi} \int_{\epsilon}^{\gamma} \frac{\tilde{q}_{-2}(\theta)}{r}+\tilde{q}_{-1}(\theta)+\tilde{q}_{0}(\theta) r+\tilde{q}_{1}(\theta) r^{2}+\tilde{q}_{2}(\theta) r^{3}+\cdots d r d \theta
$$

using polar coordinates centered at $x_{0}$ and applying a product quadrature constructed from a $3(N+2)+2$ point trapezoid rule in the variable $\theta$ and and a $\lceil N / 2\rceil+1$ Legendre quadrature rule on the interval $[0, \gamma]$. The nonintegrable term causes no difficulties since

$$
\int_{0}^{2 \pi} \frac{\tilde{q}_{-2}(\theta)}{r^{2}} d \theta=0=\frac{2 \pi}{N} \sum_{j=0}^{N-1} \frac{\tilde{q}_{-2}\left(\frac{2 \pi j}{N}\right)}{r^{2}}
$$

for any fixed $r>0$ and integer $N>1$.

The second term in (20) is more troublesome. After a chance to polar coordinates it takes on the form

$$
\int_{0}^{2 \pi} \int_{\gamma}^{\tilde{R}(\theta)} \frac{\tilde{q}_{-2}(\theta)}{r}+\tilde{q}_{-1}(\theta)+\tilde{q}_{0}(\theta) r+\tilde{q}_{1}(\theta) r^{2}+\tilde{q}_{2}(\theta) r^{3}+\cdots d r d \theta .
$$

Neither the inner integral, which for each fixed $\theta$ is of the form

$$
\int_{r_{0}}^{r_{1}} \frac{C_{-1}}{r}+C_{0}+C_{1} r+C_{2} r^{2}+\cdots d r
$$

nor the outer integral

$$
\int_{0}^{2 \pi} \tilde{q}_{-2}(\theta)[\log (\tilde{R}(\theta))-\log (\gamma)]+\tilde{q}_{-1}(\theta)[\tilde{R}(\theta)-\gamma] \tilde{q}_{0}(\theta)\left[\frac{\tilde{R}(\theta)^{2}}{2}-\frac{\gamma^{2}}{2}\right]+\cdots d \theta
$$

can be evaluated effectively using standard techniques. In the case of the inner integral this is due to the $1 / r$ term. Of course, if the exact value of $C_{-1}$ is known, then the singularity can be removed and the contribution from that term evaluated analytically. Such an approach is not always feasible and we wish our technique to 
work in the greatest generality possibly, so we assume that the exact values of the $C_{j}$ are not accessible. The poles of the function $\tilde{R}(\theta)$ and the presence of a log term make the evaluation of the outer integral difficult.
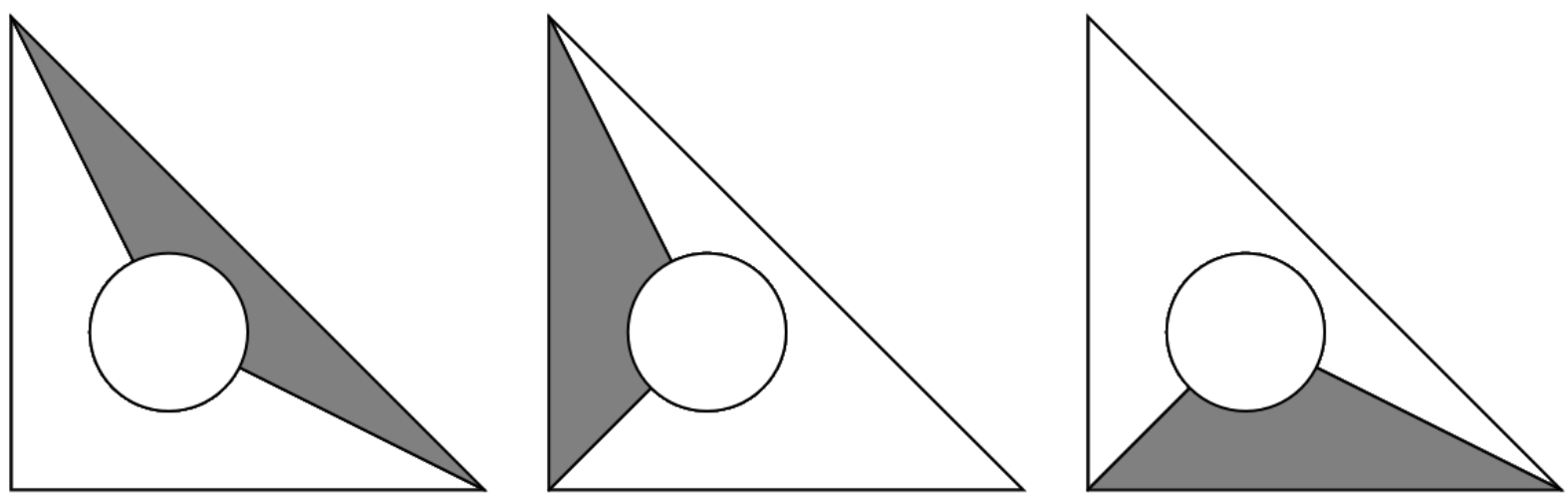

FIGURE 2. The region $T \backslash B_{\gamma}$ is further subdivided into three pieces.

The approach of this paper consists of applying tables of precomputed quadrature rules to evaluate integrals of the form (22) and (23) efficiently. Ostensibly, the quadrature rules for (22) will depend on the two parameters $r_{0}$ and $r_{1}$, but in fact due to the scale invariance of the integrands, only the ratio $r_{0} / r_{1}$ is relevant. Similarly, the function $\tilde{R}(\theta)$ in (23) initially appears to depend on 6 parameters - the coordinates of the vertices of the triangle $T$. In fact, the invariance of the integrand under scaling and rotation means that only four parameters are necessary.

A table of quadrature rules depending on four parameters would be difficult to construct. In order to reduce the number of parameters, we further decompose the region $T \backslash B_{\gamma}\left(x_{0}\right)$. More specifically, we divide it into three regions by connecting the singular point $x_{0}$ to each vertex in the fashion indicated in Figure 2. After applying a rotation and scaling, each of the resulting regions is of the form $S \backslash B_{\tilde{\gamma}}(0)$ where $S$ is a triangle with vertices $(0,0),(1,0)$ and $r_{0} e^{i \theta_{0}}$ for some $0<r_{0}<1$ and $0<\theta_{0}<\pi$; see Figure 3 for a depiction of a region of this form. Note that the constant $\gamma$ has been replaced by $\tilde{\gamma}$ due to scaling. The integral over each of the resulting regions can be written in the form

$$
\int_{0}^{\theta_{0}} \int_{\tilde{\gamma}}^{M_{r_{0}, \theta_{0}}(\theta)} \frac{\tilde{q}_{-2}(\theta)}{r}+\tilde{q}_{-1}(\theta)+\tilde{q}_{0}(\theta) r+\tilde{q}_{1}(\theta) r^{2}+\tilde{q}_{2}(\theta) r^{3}+\cdots d r d \theta,
$$

where $M_{r_{0}, \theta_{0}}(\theta)$ is the parameterization - in polar coordinates - of the line connecting the point $(0,0)$ with $r_{0} e^{i \theta_{0}}$; that is,

$$
M_{r_{0}, \theta_{0}}(\theta)=\frac{r_{0} \sin \left(\theta_{0}\right)}{r_{0} \sin \left(\theta_{0}-\theta\right)+\sin (\theta)} .
$$

Note that the outer integral in (24) now depends only on two parameters $-r_{0}$ and $\theta_{0}$.

3.3. Inner integrals. For each fixed $\theta$, the inner integral in (24) is of the form

$$
\int_{r_{0}}^{r_{1}} \frac{C_{-1}}{r}+C_{0}+C_{1} r+C_{2} r^{2}+C_{3} r^{3}+\cdots d r
$$

In order to build quadrature rules for integrals of this type which hold for a range of values of the parameters $r_{0}$ and $r_{1}$, we must first remove the dependence of the integration domain on $r_{0}$ and $r_{1}$. To that end, we apply the substitution $r=\left(r_{1}-r_{0}\right) v+r_{0}$ in order to obtain

$$
\left(r_{1}-r_{0}\right) \int_{0}^{1} \frac{C_{-1}}{\left(r_{1}-r_{0}\right) v+r_{0}}+C_{0}+C_{1}\left(r_{1}-r_{0}\right) v+r_{0}+C_{2}\left(\left(r_{1}-r_{0}\right) v+r_{0}\right)^{2}+\cdots d v .
$$

Here, the integrand ostensibly depends on both $r_{1}$ and $r_{0}$. In fact, because the form of the integrand is invariant under scaling, the integrands are only a function of the single parameter $\delta=r_{0} / r_{1}$. This can be 


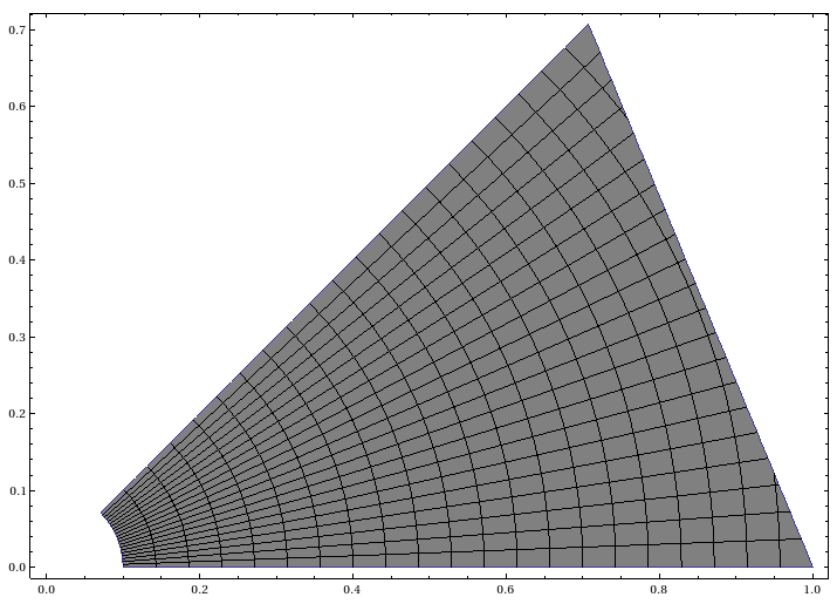

FiguRE 3. Each of three regions shown in Figure 2 can be transformed via rotation and scaling into the canonical form shown here.

easily seen by rewriting the substitution as $r=r_{1}\{(1-\delta) v+\delta\}$, which yields

$$
\int_{0}^{1} \frac{\tilde{C}_{-1}}{(1-\delta) v+\delta}+\tilde{C}_{0}+\tilde{C}_{1}[(1-\delta) v+\delta]+\tilde{C}_{2}[(1-\delta) v+\delta]^{2}+\cdots d v
$$

here, the constants $C_{j}$ have been replaced with new constants $\tilde{C}_{j}$. Next, we truncate the series expansion to obtain

$$
\int_{0}^{1} \frac{\tilde{C}_{-1}}{(1-\delta) v+\delta}+\tilde{C}_{0}+\tilde{C}_{1}[(1-\delta) v+\delta]+\tilde{C}_{2}[(1-\delta) v+\delta]^{2}+\cdots \tilde{C}_{N+1}[(1-\delta) v+\delta]^{N+1} d v .
$$

Quadrature for the integrals (26) will obtain order $N$ algebraic accuracy when applied to (25). In order to construct a quadrature rule which can be applied to integrals of the form (26) with $\delta$ any real in the interval $[a, b] \subset(0,1]$, we let $\delta_{1}, \delta_{2}, \ldots, \delta_{16}$ denote the nodes of the 16-point Legendre quadrature on the interval $[a, b]$, fix an integer $N$ and apply the algorithm of Section 1 to all functions of the form

$$
\left[\left(1-\delta_{j}\right) v+\delta_{j}\right]^{i}, \quad i=-1, \ldots, N+1, \quad j=1, \ldots, 16 .
$$

For the experiments of this paper, rules of order $N=4, N=8, N=12$ and $N=16$ were constructed. For each order, 23 of these quadrature rules - each applying to a different range of values of the parameter $\delta$ were constructed. Presenting the length of each of the 92 rules in this work would take a prohibitive amount of space. We settle for showing the length of selected rules in Table 2.

\begin{tabular}{ccccc}
$\delta$ range & order 4 & order 8 & order 12 & order 16 \\
\hline $1.00 \times 10^{-14}-1.00 \times 10^{-13}$ & 16 & 17 & 20 & 21 \\
$1.00 \times 10^{-07}-1.00 \times 10^{-06}$ & 15 & 17 & 19 & 21 \\
$1.00 \times 10^{-03}-1.00 \times 10^{-04}$ & 15 & 17 & 19 & 21 \\
$1.00 \times 10^{-01}-2.00 \times 10^{-01}$ & 10 & 11 & 13 & 15 \\
$0.90 \times 10^{-00}-1.00 \times 10^{-00}$ & 5 & 6 & 7 & 9
\end{tabular}

TABLE 2. The length of selected quadrature rules of Section 3.3. 
3.4. Outer integrals. After applying the substitution $u=\theta_{0} \theta$, which removes the dependence of the integration domain on the parameter $\theta_{0}$, the outer integral in (24) can be written as

$$
\begin{array}{r}
\theta_{0} \int_{0}^{1} \tilde{q}_{-2}\left(\theta_{0} u\right)\left[\log \left(M_{r_{0}, \theta_{0}}\left(\theta_{0} u\right)\right)-\log (\tilde{\gamma})\right]+\tilde{q}_{-1}\left(\theta_{0} u\right)\left[M_{r_{0}, \theta_{0}}(\theta)-\tilde{\gamma}\right]+ \\
\tilde{q}_{0}\left(\theta_{0} u\right)\left[\frac{M_{r_{0}, \theta_{0}}^{2}\left(\theta_{0} u\right)}{2}-\frac{\tilde{\gamma}^{2}}{2}\right]+\cdots d u .
\end{array}
$$

After truncation and a slight rearrangement, it takes on the form

$$
\theta_{0} \int_{0}^{1} \tilde{q}_{-2}\left(\theta_{0} u\right) \log \left(M_{r_{0}, \theta_{0}}\left(\theta_{0} u\right)\right)+\tilde{q}_{-1}\left(\theta_{0} u\right) M_{r_{0}, \theta_{0}}\left(\theta_{0} u\right)+\cdots+\tilde{q}_{N+1}\left(\theta_{0} u\right) \frac{M_{r_{0}, \theta_{0}}^{(N+1)}\left(\theta_{0} u\right)}{N+1}+p\left(\theta_{0} u\right) d u,
$$

where $p(\theta)$ is a trigonometric polynomial of order $3(N+2)+2$. In order to construct a quadrature rule for integrals of this form which hold for $\theta_{0}$ in $[a, b] \subset(0,2 \pi)$ and $r_{0}$ in $[c, d] \subset(0,1)$, we first let

$$
\theta_{1}, \ldots, \theta_{16}, \text { and } r_{1}, \ldots, r_{16}
$$

denote the nodes of the 16-point Legendre quadrature on the intervals $[a, b]$ and $[c, d]$, respectively. Then we apply the algorithm of Section 1 to the functions

$$
M_{r_{0}, \theta_{0}}\left(\theta_{0} u\right)^{j} \cos (i \theta), \quad M_{r_{0}, \theta_{0}}\left(\theta_{0} u\right)^{j} \sin (i \theta) \quad j=0, \ldots, N+1, \quad i=0, \ldots, 3(j+1)+2,
$$

and

$$
\cos \left(i \theta_{0} u\right), \quad \sin \left(i \theta_{0} u\right) \quad i=0, \ldots, 3(N+2)+2 .
$$

Quadrature rules were constructed for orders $N=4, N=8, N=12$ and $N=16$. The parameter $r_{0}$ was partitioned For each of those orders, quadrature rules for each pair of 9 intervals of $r_{0}$ and 14 of $\theta_{0}$ were It is clearly not possible to present the lengths of all 504 quadrature rules. Instead, we present the lengths of selected rules in Table 3 .

\begin{tabular}{cccccc}
$r_{0}$ range & $\theta_{0}$ range & order 4 & order 8 & order 12 & order 16 \\
\hline $1.00 \times 10^{-06}-1.00 \times 10^{-05}$ & $1.00 \times 10^{-06}-1.00 \times 10^{-05}$ & 36 & 44 & 50 & 53 \\
$1.00 \times 10^{-06}-0.00 \times 10^{-05}$ & $3.14-3.14159$ & 70 & 81 & 90 & 100 \\
$1.00 \times 10^{-04}-1.00 \times 10^{-03}$ & $0.10-0.25$ & 38 & 44 & 49 & 55 \\
$0.10-0.30$ & $0.50-1.00$ & 26 & 31 & 34 & 37 \\
$0.90-1.00$ & $3.14-3.14159$ & 71 & 85 & 99 & 100
\end{tabular}

TABLE 3. The length of selected quadrature rules of Section 3.4.

\section{Numerical EXPERIMENTS}

We now describe numerical experiments conducted to measure the performance of the approach of this article. All code was written in Fortran 77 and compiled with the Intel Fortran Compiler version 12.1. The experiments were carried out on an workstation equipped with 12 Intel Xeon processor cores running at 3.47 GHz and $192 \mathrm{~GB}$ of RAM. Throughout this section we use the notation for various integral operators established in (5) and (6) of the introduction.

In several experiments of this section integral equation formulations which are susceptible to spurious resonances were utilized. For instance, the integral operator $1 / 2 I+D_{k}$ appearing in Section 4.1 will have a nontrivial nullspace for certain values of the wavenumber $k$. All experiments were designed so that the wavenumbers $k$ chosen lead to uniquely solvable integral equations.

4.1. A comparison with fixed-order quadrature rules. In most integral equation solvers, Gaussian quadratures are used to evaluate the singular integrals of the form

$$
\int_{0}^{2 \pi} \int_{0}^{R(\theta)} q_{-1}(\theta)+q_{0}(\theta) r+q_{1}(\theta) r^{2}+q_{2}(\theta) r^{3}+\cdots d r d \theta
$$

which arise during the discretization of weakly singular integral operators. The purpose of this set of experiments was to compare the effectiveness of Gaussian quadratures with the precomputed quadrature rules of this paper in a simple case, although admittedly artificial case. 
The interior Dirichlet problem

$$
\begin{aligned}
\Delta u+k^{2} u & =0 & & \text { in } \Omega \\
u & =g & & \text { on } \partial \Omega
\end{aligned}
$$

was considered on the domain $\Omega$ bounded by the surface parameterized via

$$
r(s, t)=\left(\begin{array}{c}
(2+0.2 \cos (s)) \sin (t) \\
(2+0.2 \cos (s)) \cos (t) \\
0.2 \sin (t)
\end{array}\right), \quad 0 \leq s, t<2 \pi .
$$

The wavenumber $k$ was taken to be 1 and the function $g$ was defined by

$$
g(q)=\frac{1}{4 \pi} \iint_{S} \frac{\exp (i k|q-p|)}{|q-p|} d s(p)
$$

where $S$ denotes the boundary of the ball of radius 1 centered at the point $(10,0,10)$. This boundary value problem can be reformulated as

$$
\frac{1}{2} \sigma(q)+D_{k} \sigma(q)=g(q), \quad(q \in \partial \Omega)
$$

\begin{tabular}{|c|c|c|c|c|c|c|c|}
\hline \multirow[b]{2}{*}{$N_{\text {tris }}$} & \multirow[b]{2}{*}{$N$} & \multicolumn{2}{|c|}{12 order precomputed } & \multicolumn{2}{|c|}{ Gaussian $(n=50)$} & \multicolumn{2}{|c|}{ Gaussian $(n=100)$} \\
\hline & & $N_{\text {avg }}$ & $E_{\text {solution }}$ & $N_{\text {avg }}$ & $E_{\text {solution }}$ & $N_{\text {avg }}$ & $E_{\text {solution }}$ \\
\hline 20 & 2240 & 2918 & $7.72 \times 10^{-05}$ & 1050 & $7.76 \times 10^{-05}$ & 2100 & $7.72 \times 10^{-05}$ \\
\hline 80 & 8960 & 2909 & $3.98 \times 10^{-10}$ & 1050 & $1.97 \times 10^{-05}$ & 2100 & $1.05 \times 10^{-06}$ \\
\hline \multirow{2}{*}{320} & 35840 & 2908 & $9.75 \times 10^{-14}$ & 1050 & $5.14 \times 10^{-06}$ & 2100 & $2.37 \times 10^{-07}$ \\
\hline & & \multicolumn{2}{|c|}{ Gaussian $(n=200)$} & \multicolumn{2}{|c|}{ Gaussian $(n=400)$} & \multicolumn{2}{|c|}{ Gaussian $(n=1000)$} \\
\hline$N_{\text {tris }}$ & $N$ & $N_{\text {avg }}$ & $E_{\text {solution }}$ & $N_{\text {avg }}$ & $E_{\text {solution }}$ & $N_{\text {avg }}$ & $E_{\text {solution }}$ \\
\hline 20 & 2240 & 4200 & $7.72 \times 10^{-05}$ & 8400 & $7.72 \times 10^{-05}$ & 21000 & $7.72 \times 10^{-05}$ \\
\hline 80 & 8960 & 4200 & $1.55 \times 10^{-08}$ & 8400 & $3.31 \times 10^{-10}$ & 21000 & $3.99 \times 10^{-10}$ \\
\hline 320 & 35840 & 4200 & $4.02 \times 10^{-09}$ & 8400 & $4.05 \times 10^{-11}$ & 21000 & $4.99 \times 10^{-14}$ \\
\hline
\end{tabular}

by introducing the representation

$$
u(q)=D_{k} \sigma(q)
$$

of the solution $u$.

TABLE 4. The results of the experiments of Section 4.1.

In each experiment, the integral equation (30) was discretized repeatedly by applying the Nyström approach of Section 2 with progressively finer decompositions of $\partial \Omega$. An 112 point 12th order discretization quadrature was applied to each surface element. The method used to evaluate singular integrals was varied from experiment to experiment. In the first experiment, tensor products of 12th order precomputed quadratures of Section 3.3 and Section 3.4 were applied to the singular integrals of the form (28) which arose. In the later experiments, a fixed Legendre quadrature of length 7 (which integrates polynomials of order 0 through 13) was applied to the inner integrals and a second piecewise Gaussian quadrature was applied to the outer integrals. The piecewise quadrature consisted of 3 length $n$ Legendre rules placed on the three intervals of $[0,2 \pi]$ on which $R(\theta)$ is smooth, so a total of $21 n$ Gaussian nodes were used. Each discretized integral equation was inverted via the GMRES iterative scheme - iterations were terminated when residuals fell below $1.0 \times 10^{-13}$ - and the relative error

$$
\left|\frac{u\left(q_{0}\right)-g\left(q_{0}\right)}{g\left(q_{0}\right)}\right|
$$

where $q_{0}$ is the point $(2,0,0)$, was calculated.

Table 4 shows the results. There, the headings indicate which quadratures were used to evaluate singular integrals, $N_{\text {tris }}$ refers to the number of triangles into which the parameterization domain was subdivided; $N$ is the number of discretization nodes on the surface $\partial \Omega ; N_{\text {avg }}$ gives the average size of the quadratures used 
to evaluate singular integrals; and $E_{\text {solution }}$ is the relative error (32). Clearly, this is a case where the use of Gaussian rules reduces the rate of convergence which would otherwise be obtained by the approximation of the solution using 12th order piecewise polynomials. That is, until very large tensor product Gaussian quadratures are applied. On the other hand, 12th order convergence is obtained when the precomputed rules of this paper are utilized.

4.2. Tangential derivatives on a deformed torus. The purpose of this set of experiments was to test the accuracy of the quadrature rules described in Section 3. The setting was the compact domain $\Omega$ bounded by the surface parameterized via

$$
r(s, t)=\left(\begin{array}{c}
(2+\cos (s)) \sin (t) \\
(2+\cos (s)) \cos (t) \\
\sin (s)(1+0.20 \cos (4 t))
\end{array}\right), \quad 0 \leq s, t<2 \pi .
$$

Note that the surface $\partial \Omega$ is not a surface of revolution; this is an important point since simpler discretization mechanisms are available in that setting (see, for instance, [23]). Three views of the surface $\partial \Omega$ are shown in Figure 4.
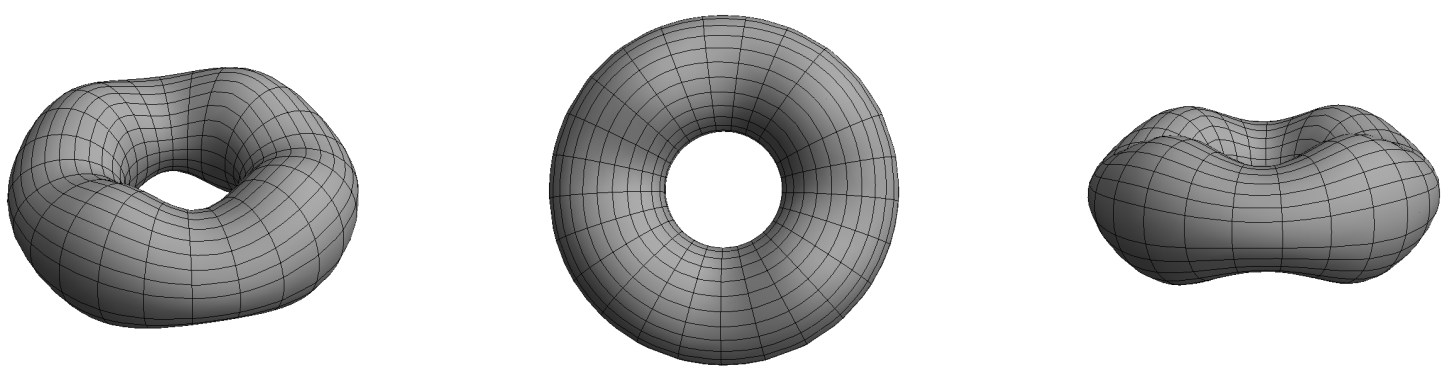

FiguRE 4. Three views of the surface of Section 4.2 .

The interior Neumann problem

$$
\begin{aligned}
\Delta u+k^{2} u & =0 & & \text { in } \Omega \\
\frac{\partial u}{\partial \eta} & =g & & \text { on } \partial \Omega,
\end{aligned}
$$

where $\frac{\partial}{\partial \eta}$ denotes differentiation with respect to the outward-pointing unit normal vector, can be reformulated as the integral equation

by introducing the representation

$$
-\frac{1}{2} \sigma(q)+D_{k}^{*} \sigma(q)=g(q), \quad(q \in \partial \Omega),
$$

$$
u(q)=S_{k} \sigma(q) .
$$

Once the solution of (34) has been obtained in the form (36), the tangential derivative $\nabla u(q) \cdot \frac{\partial r}{\partial s}(q)$ at a point $q$ on the boundary $\partial \Omega$ is given by the formula

$$
\nabla u(q) \cdot \frac{\partial r}{\partial s}(q)=\lim _{\epsilon \rightarrow 0} \frac{1}{4 \pi} \iint_{\partial \Omega \backslash B_{\epsilon}(q)}\left(\frac{(q-p)}{|q-p|^{3}} \cdot \frac{\partial r}{\partial s}(q)\right) \exp (i k|q-p|)(1-i k|q-p|) \sigma(p) d s(p) .
$$

This integral operator is, of course, only defined in the sense of the Cauchy principal value. For these experiments, the wavenumber $k$ was taken to be 1 and the right-hand side $g$ was taken to be the normal derivative of the function

$$
f(q)=\iint_{\partial B_{1}(10,0,10)} \frac{\exp (i k|q-p|)}{|q-p|} d s(p)
$$

where $B_{r}(q)$ once again denotes the boundary of the ball of radius $r$ centered at the point $q$. The function $f$ is, of course, the unique solution of the boundary value problem (34); considering a problem with a known solutions allows us to explicitly compute the error obtained by the solver. Note that the the integral operator 
$1 / 2 I+D_{k}^{*}$ will have a nontrivial nullspace for certain values of the wavenumber $k$ but the experiments of this section were designed to avoid this difficulty.

In each of the experiments of this section, the integral equation (35) was discretized using the Nyström method detailed in Section 2 with singular integrals evaluated via the quadratures of Section 3. Decompositions of the surface $\partial \Omega$ were obtained by triangulating the parameterization domain $[0,2 \pi] \times[0,2 \pi]$. The resulting system of linear equations was solved via the GMRES iterative algorithm, which was terminated after the residuals fell below $1.0 \times 10^{-13}$. The $L^{2}\left(\partial B_{0.1}(2,0,0)\right)$ relative error

$$
\left(\iint_{\partial B_{0.1}(2,0,0)}|f(q)|^{2} d s(q)\right)^{-1 / 2}\left(\iint_{\partial B_{0.1}(2,0,0)}\left|f(q)-S_{k} \sigma(q)\right|^{2} d s(q)\right)^{1 / 2}
$$

was computed in order to measure the accuracy of the obtained approximation

\begin{tabular}{rrrccrrrc}
\multicolumn{9}{c}{ 4th order } \\
$N_{\text {tris }}$ & $N$ & $N_{\text {avg }}$ & $E_{\text {solution }}$ & $E_{\text {tangent }}$ & $N$ & $N_{\text {avg }}$ & $E_{\text {solution }}$ & $E_{\text {tangent }}$ \\
\hline 4 & 72 & 1567 & $7.48 \times 10^{-02}$ & $3.45 \times 10^{-01}$ & 208 & 2096 & $2.46 \times 10^{-03}$ & $8.62 \times 10^{-02}$ \\
16 & 288 & 1515 & $1.99 \times 10^{-03}$ & $7.56 \times 10^{-02}$ & 832 & 2079 & $2.51 \times 10^{-05}$ & $4.51 \times 10^{-03}$ \\
64 & 1152 & 1523 & $9.24 \times 10^{-05}$ & $5.72 \times 10^{-03}$ & 3328 & 2072 & $4.86 \times 10^{-09}$ & $7.03 \times 10^{-05}$ \\
256 & 4608 & 1524 & $6.79 \times 10^{-08}$ & $2.49 \times 10^{-04}$ & 13312 & 2075 & $1.01 \times 10^{-11}$ & $4.26 \times 10^{-07}$ \\
1024 & 18432 & 1526 & $3.96 \times 10^{-10}$ & $8.64 \times 10^{-06}$ & 53248 & 2073 & $1.77 \times 10^{-13}$ & $1.28 \times 10^{-09}$ \\
4096 & 73728 & 1526 & $8.66 \times 10^{-12}$ & $2.74 \times 10^{-07}$ & 212992 & 2073 & $2.80 \times 10^{-13}$ & $6.24 \times 10^{-12}$ \\
16384 & 294912 & 1526 & $3.10 \times 10^{-12}$ & $8.60 \times 10^{-09}$ & & & &
\end{tabular}

\begin{tabular}{rrrccrrrc} 
& \multicolumn{4}{c}{12 th order } & \multicolumn{3}{c}{16 th order } \\
$N_{\text {tris }}$ & $N$ & $N_{\text {avg }}$ & $E_{\text {solution }}$ & $E_{\text {tangent }}$ & $N$ & $N_{\text {avg }}$ & $E_{\text {solution }}$ & $E_{\text {tangent }}$ \\
\hline 4 & 448 & 2744 & $7.98 \times 10^{-04}$ & $2.02 \times 10^{-02}$ & 768 & 3529 & $2.80 \times 10^{-05}$ & $5.25 \times 10^{-03}$ \\
16 & 1792 & 2713 & $7.03 \times 10^{-08}$ & $2.02 \times 10^{-04}$ & 3072 & 3492 & $1.19 \times 10^{-08}$ & $2.57 \times 10^{-05}$ \\
64 & 7168 & 2710 & $1.23 \times 10^{-10}$ & $1.70 \times 10^{-06}$ & 12288 & 3487 & $2.49 \times 10^{-12}$ & $5.34 \times 10^{-08}$ \\
256 & 28672 & 2713 & $3.84 \times 10^{-13}$ & $1.35 \times 10^{-09}$ & 49152 & 3491 & $2.61 \times 10^{-13}$ & $8.21 \times 10^{-12}$ \\
1024 & 114688 & 2713 & $3.41 \times 10^{-13}$ & $8.39 \times 10^{-12}$ & 196608 & 3492 & $1.45 \times 10^{-14}$ & $1.23 \times 10^{-12}$
\end{tabular}

TABle 5. Results of the experiments described in Section 4.2.

The approach of Section 2 was then applied to discretize the principal value integral operator appearing in (37). The quadratures of Section 3 were used to evaluate singular integrals. The discrete operator was then applied to the computed charge distribution in order to approximate the tangential derivative $\nabla u(q) \cdot \frac{\partial r}{\partial s}(q)$ on the boundary $\partial \Omega$. The relative $L^{2}(\partial \Omega)$ accuracy of the obtained tangential derivative was calculated by comparison with the tangential derivative of the function $f(q)$ on $\partial \Omega$, which is known and can be computed directly. Table 5 displays the results. There, $N_{\text {tris }}$ denotes the number of triangles into which the parameterization domain $[0,2 \pi] \times[0,2 \pi]$ was subdivided; $N$ denotes the number of discretization nodes; $N_{\mathrm{avg}}$ is the average size of the quadratures used to evaluate singular integrals; $E_{\text {solution }}$ denotes the relative error (38); and $E_{\text {tangent }}$ denotes the relative $L^{2}(\partial \Omega)$ error in the computed tangential derivative of the solution $u$.

In the case of 8 th, 12 th and 16th order quadratures, approximately $12-13$ digits of precision were obtained for the solution of the integral equations and tangential derivatives were computed to around $11-12$ digits of precision. Were we unable to obtain the same level of accuracy using 4th order quadrature formula only because these experiments were carried out without the aid of an accelerated solver (e.g... a multipole code or fast direct solver) and this made experiments involving large discretizations prohibitively expensive to conduct. 
4.3. Tangential derivatives on a heart-shaped domain. In these experiments, the exterior Neumann problem

$$
\begin{array}{rlrl}
\Delta u+k^{2} u=0 & & \text { in } \Omega^{c} \\
\frac{\partial u}{\partial \eta}=g & & \text { on } \partial \Omega \\
\frac{\partial u}{\partial r}-i k u=O\left(\frac{1}{r}\right)
\end{array}
$$

was considered on the heart-shaped domain $\Omega$ shown in Figure 5. The boundary $\partial \Omega$ is described by the parameterization

$$
r(s, t)=\left(\begin{array}{c}
\cos (s) \cos (t)+\cos ^{2}(s) \sin ^{2}(t) \\
\cos (s) \sin (t) \\
\sin (s)
\end{array}\right), \quad 0 \leq s, t<2 \pi,
$$

although for the purposes of producing decompositions of the surface $\partial \Omega$ a parameterization obtained via projection onto a cube was used rather than this function $r(s, t)$. The problem (39) can be reformulated as
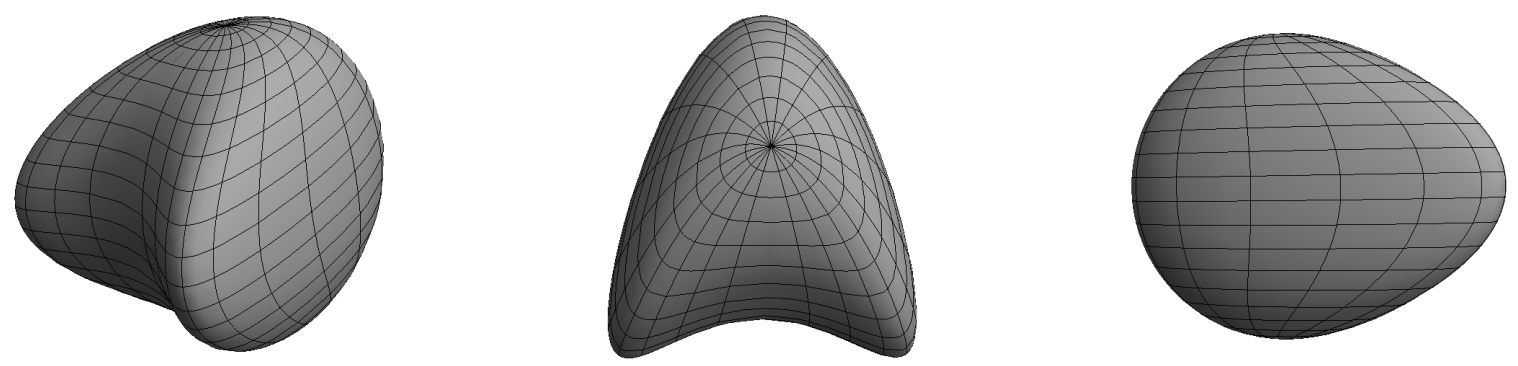

FiguRE 5. Three views of the surface of Section 4.3.

the integral equation

by introducing the representation

$$
\frac{1}{2} \sigma(q)+D_{k}^{*} \sigma(q)=g(q), \quad(q \in \partial \Omega)
$$

$$
u(q)=S_{k} \sigma(q) .
$$

The experiments of the preceding section were repeated in this setting with minor changes: the wavenumber $k$ was taken to be $\pi$; the right-hand side $g$ was taken to be the normal derivative of the function

$$
f(q)=\iint_{\partial B_{0.1}(0,0,0)} \frac{\exp (i k|q-p|)}{|q-p|} d s(p) ;
$$

and the relative error in the solution was measured by computing

$$
\left(\iint_{\partial B_{1}(10,0,10)}|f(q)|^{2} d s(q)\right)^{-1 / 2}\left(\iint_{\partial B_{1}(10,0,10)}\left|f(q)-S_{k} \sigma(q)\right|^{2} d s(q)\right)^{1 / 2} .
$$

Table 6 shows the results. Once again, we see that high-accuracy and high-order convergence can be obtained using the precomputed quadrature rules of this paper.

4.4. Electromagnetic scattering from an ellipsoid. The boundary value problem

$$
\begin{aligned}
\nabla \times E=i k H & \text { in } \Omega^{c} \\
\nabla \times H=-i k E & \text { in } \Omega^{c} \\
\nabla \cdot E=0 & \text { in } \Omega^{c} \\
\nabla \cdot H=0 & \text { in } \Omega^{c} \\
\eta \times E=0 & \text { on } \partial \Omega
\end{aligned}
$$




\begin{tabular}{rrrccrrrc} 
& \multicolumn{4}{c}{12 th order } & \multicolumn{3}{c}{16 th order } \\
$N_{\text {tris }}$ & $N$ & $N_{\text {avg }}$ & $E_{\text {solution }}$ & $E_{\text {tangent }}$ & $N$ & $N_{\text {avg }}$ & $E_{\text {solution }}$ & $E_{\text {tangent }}$ \\
\hline 48 & 5376 & 2757 & $1.69 \times 10^{-09}$ & $8.33 \times 10^{-06}$ & 9216 & 3539 & $2.82 \times 10^{-11}$ & $1.86 \times 10^{-07}$ \\
192 & 21504 & 2750 & $3.84 \times 10^{-13}$ & $9.07 \times 10^{-09}$ & 36864 & 3534 & $1.75 \times 10^{-13}$ & $6.33 \times 10^{-11}$ \\
768 & 86016 & 2750 & $6.53 \times 10^{-13}$ & $5.67 \times 10^{-11}$ & & & &
\end{tabular}

TABLE 6. Results of the experiments of Section 4.3.

arises from the scattering of time-harmonic waves from a perfect conductor $\Omega$. Here, $\eta$ is the outward-pointing unit normal vector to $\partial \Omega$ and $E$ and $H$ represent the total electric and magnetic fields. That is,

$$
\begin{aligned}
& E=E_{\text {in }}+E_{\text {scat }} \\
& H=H_{\text {in }}+H_{\text {scat }},
\end{aligned}
$$

where $E_{\text {in }}$ and $H_{\text {in }}$ are incident fields and $E_{\text {scat }}$ and $H_{\text {scat }}$ denote scattered fields of interest. The augmented electric field integral equation (see [21] for details) is obtained by inserting the representation

$$
E_{\text {scat }}=i k A-\nabla \phi
$$

where

$$
A(q)=\frac{1}{4 \pi} \iint_{\partial \Omega} \frac{e^{i k|q-p|}}{|q-p|} J(p) d s(p)
$$

and

$$
\phi(q)=\frac{1}{4 \pi} \iint \frac{e^{i k|q-p|}}{|q-p|} \rho(p) d s(p),
$$

into (40). Specifically, a solution to (42) of the form (41) can be obtained by solving the system of integral equations

$$
\begin{gathered}
0=\eta \times E=\eta \times E_{\text {in }}+\eta \times E_{\text {scat }} \\
\rho=\eta \cdot E=\eta \cdot E_{\text {in }}+\eta \cdot E_{\text {scat }} .
\end{gathered}
$$

The unknowns in (42) are the surface current $J$ and charge distribution $\rho$, both of which are given on $\partial \Omega$. The operators in this system are defined only in the sense of Cauchy principal values.

In the experiments described in this section, the domain $\Omega$ was taken to be the exterior of the ellipsoid defined by the equation

$$
x^{2}+y^{2}+\frac{z^{2}}{4}=1
$$

Three views of the surface $\partial \Omega$ are shown in Figure 6 . This surface was parameterized by projecting it onto
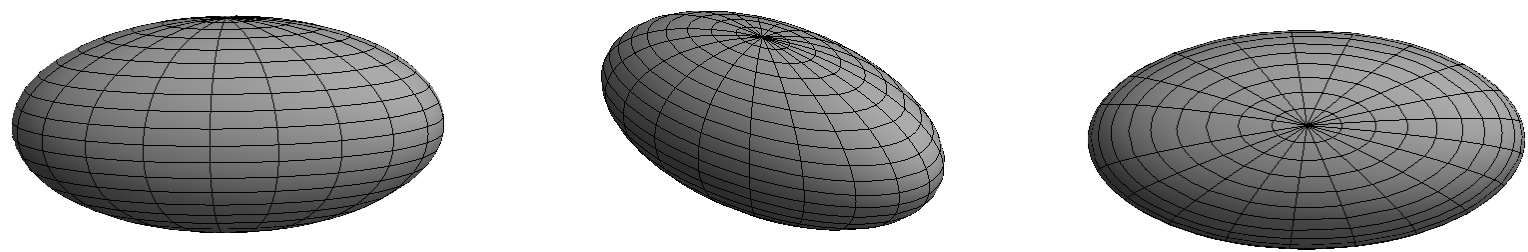

Figure 6. Three views of the domain of Section 4.4.

the boundary of the cube $[-1,1]^{3}$; for instance, the portion of $\partial \Omega$ shown in Figure 7 was parameterized over the top face of the cube via the mapping

$$
\left(\begin{array}{c}
s \\
t \\
1
\end{array}\right) \rightarrow \frac{1}{\sqrt{s^{2}+t^{2}+1}}\left(\begin{array}{c}
s / 10 \\
t \\
1
\end{array}\right)
$$


The wavenumber $k$ was taken to be 1 and the incoming electric field was the function

$$
E_{\text {in }}(q)=G_{e}\left(q, p_{0}\right) v
$$

where $G_{e}(q, p)$ is the dyadic electric Green's function

$$
\begin{gathered}
G_{e}(q, p)=\frac{i k}{4 \pi}\left[I+\frac{\nabla \nabla}{k^{2}}\right] \frac{e^{i k|q-p|}}{|q-p|}, \\
p_{0}=\frac{1}{10}\left(\begin{array}{c}
2 \\
-3 \\
1
\end{array}\right) \quad \text { and } \quad v=\left(\begin{array}{c}
1 \\
1 \\
1
\end{array}\right) .
\end{gathered}
$$

In each experiment, the parameterization domain of the ellipsoid was triangulated and the integral equation (42) discretized using the approach of this paper. The resulting linear system was solved using the GMRES algorithm; iterations were terminated when the residual fell below $1.0 \times 10^{-13}$ in magnitude. The true values of the electromagnetic field $E$, the magnetic field $H$ and the quantities $\eta \cdot H$ are known by the extinction theorem. We compared them with the obtained values on the sphere $S$ of radius 50 centered at 0 . The quantity $\eta \cdot H$ was computed on the surface $\partial \Omega$. The computation of $\eta \cdot H$ involves the evaluation of a principal value integral operator.

Table 7 displays the results. There, $N_{\text {tris }}$ is the numbers of triangles into which the parameterization domain was subdivided; $E$ is the relative $L^{2}(\partial \Omega)$ error in the electric field $E$ on the sphere $S ; H$ is the relative $L^{2}(S)$ error on the sphere $S$; and $\eta \cdot H$ is the relative $L^{2}(S)$ error in the quantity $\eta \cdot H$.

\begin{tabular}{rcccccc}
\multicolumn{5}{c}{ 4th order } \\
$N_{\text {tris }}$ & $E$ & $H$ & $\eta \cdot H$ & $E$ & 8th order \\
\hline 12 & $8.71 \times 10^{-02}$ & $6.83 \times 10^{-02}$ & $2.17 \times 10^{-00}$ & $1.02 \times 10^{-02}$ & $7.08 \times 10^{-03}$ & $1.94 \times 10^{-00}$ \\
48 & $2.82 \times 10^{-03}$ & $2.22 \times 10^{-03}$ & $4.05 \times 10^{-01}$ & $4.42 \times 10^{-05}$ & $3.72 \times 10^{-05}$ & $8.33 \times 10^{-02}$ \\
192 & $1.33 \times 10^{-04}$ & $1.09 \times 10^{-04}$ & $6.19 \times 10^{-02}$ & $8.11 \times 10^{-06}$ & $6.75 \times 10^{-07}$ & $1.89 \times 10^{-03}$ \\
432 & $1.90 \times 10^{-05}$ & $1.47 \times 10^{-05}$ & $1.12 \times 10^{-02}$ & $4.51 \times 10^{-08}$ & $3.46 \times 10^{-08}$ & $8.43 \times 10^{-05}$ \\
768 & $2.10 \times 10^{-06}$ & $1.65 \times 10^{-06}$ & $3.21 \times 10^{-03}$ & & & \\
1200 & $1.42 \times 10^{-07}$ & $1.07 \times 10^{-07}$ & $1.21 \times 10^{-03}$ & & &
\end{tabular}

\begin{tabular}{rcccccc}
\multicolumn{3}{c}{12 th order } & \multicolumn{3}{c}{16 th order } \\
$N_{\text {tris }}$ & $E$ & $H$ & $\eta \cdot H$ & $E$ & $H$ & $\eta \cdot H$ \\
\hline 12 & $1.33 \times 10^{-03}$ & $1.11 \times 10^{-03}$ & $1.70 \times 10^{-00}$ & $1.45 \times 10^{-04}$ & $1.16 \times 10^{-04}$ & $8.18 \times 10^{-01}$ \\
48 & $3.03 \times 10^{-06}$ & $2.34 \times 10^{-06}$ & $1.14 \times 10^{-02}$ & $1.83 \times 10^{-07}$ & $1.44 \times 10^{-07}$ & $1.76 \times 10^{-03}$ \\
192 & $1.47 \times 10^{-08}$ & $1.19 \times 10^{-08}$ & $4.60 \times 10^{-05}$ & $2.09 \times 10^{-10}$ & $1.83 \times 10^{-10}$ & $3.36 \times 10^{-06}$
\end{tabular}

TABLE 7. The results of the experiments of Section 4.4.
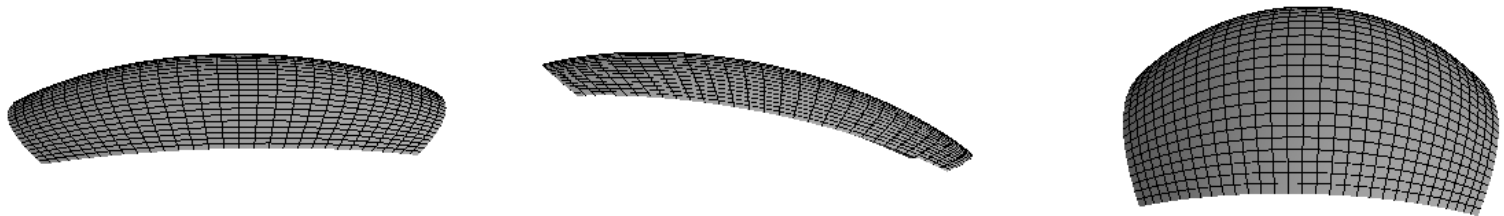

FiguRE 7. The portion of the ellipsoid of Section 4.4 which is parameterized over the top face of the cube $[-1,1]^{3}$.

Using 16th order quadratures, approximately 9 digits of precision was obtained for the electric field $E$ and magnetic field $H$ and 5 for the quantity $\eta \cdot H$. The code in these experiments was unaccelerated and as a result running these experiments with larger discretizations is prohibitively expensive. Coupling the discretization 
approach of this paper with an appropriate fast solver should enable larger-scale experiments and higher accuracy. Doing so, however, is beyond the scope of this work.

\section{Conclusions}

The fast and accurate evaluation of singular integrals is one of the principal challenges in integral equation methods. Both the evaluation of singular integrals given over curved surfaces and the evaluation of expressions involving nonintegrable kernels present severe difficulties for currently available methods. This paper introduces what the authors believe to be one of most accurate methods for simultaneously addressing both situations. Moreover, it requires considerably less analytic information in order to achieve high-order convergence than other comparable schemes of which the authors are aware. For instance, the solver described in [22] achieves high-order convergence for weakly singular integral operators, but at the price of requiring that a smooth partition of unity for the surface be given.

Although the scheme of this paper is reasonably efficient, there are several obvious mechanisms for reducing the size of the resulting quadrature formulae. Perhaps the most straightforward is to generalize the approach of Section 1 to two-dimensions in order to replace formulae composed of products of one-dimensional quadrature rules with true two-dimensional quadratures. Another possibility is to avoid the decomposition of the integration domain. Each of these refinements would reduce the size of the quadrature rules; the combination of both might have a significant impact. The computation of efficient two-dimensional quadrature rules is a significant computational challenge, however, and it is a subject of ongoing research by the authors.

\section{Acknowledgments}

The authors would like to thank the referee for carefully reviewing the manuscript and making many helpful suggestions. We would also like to thank Leslie Greengard and John Strain for useful discussions and Adrianna Gillman for supplying the parameterization of the domain of Section 4.3.

James Bremer was supported by a fellowship from the Alfred P. Sloan Foundation and by the Office of Naval Research under contract N00014-12-1-0117. Zydrunas Gimbutas was supported by Air Force Office of Scientific Research under NSSEFF Program Award FA9550-10-1-0180 and by the National Science Foundation under grant DMS-0934733.

\section{REFERENCES}

[1] Atkinson, K. E. The Numerical Solution of Integral Equations of the Second Kind. Cambridge University Press, Cambridge, 1997.

[2] Barnett, A., And Greengard, L. A new integral representation for quasi-periodic fields and its application to twodimensional band structure calculations. Journal of Computational Physics 229 (2010), 6898-6914.

[3] Bremer, J. On the Nyström discretization of integral operators on planar domains with corners. Applied and Computational Harmonic Analysis (to appear).

[4] Bremer, J., And Gimbutas, Z. A Nyström method for weakly singular integral operators on surfaces. Journal of Computational Physics 231 (2012), 4885-4903.

[5] Bremer, J., Gimbutas, Z., and Rokhlin, V. A nonlinear optimization procedure for generalized Gaussian quadratures. SIAM Journal of Scientific Computing 32 (2010), 1761-1788.

[6] Chernov, A., von Petersdorff, T., And Schwab, C. Exponential convergence of $h p$ quadrature for integral operators with Gervey kernels. ESAIM: Mathematical Modelling and Numerical Analysis 45 (2011), 387-422.

[7] Coifman, R. R., And Meyer, Y. Wavelets: Calderón-Zygmund and Multilinear Operators. Cambridge University Press, Cambridge, 1997.

[8] Colton, D., And Kress, R. Integral Equation Methods in Scattering Theory. John Wiley \& Sons, New York, 1983.

[9] Epstein, C. L., And Greengard, L. Debye sources and the numerical solution of the time harmonic Maxwell equations. Communications on Pure and Applied Mathematics 63 (2010), 413-463.

[10] HackBusch, W. Integral Equations: theory and numerical treatment. Birkhäuser, Berlin, 1995.

[11] Jackson, J. D. Classical Electrodynamics, 3rd ed. Wiley, New York, 1998.

[12] Kress, R. Integral Equations. Springer-Verlag, New York, 1999.

[13] Mikhlin, S. Integral Equations. Pergamon Press, New York, 1957.

[14] Morse, P. M., And Feshbach, H. Methods of Mathematical Physics. Feshbach Publishing, New York, 1998.

[15] NÉDÉLEC, J.-C. Acoustic and Electromagnetic Equations: Integral Representations for Harmonic Functions. Springer, New York, 2012.

[16] Rokhlin, V. Solution of acoustic scattering problems by means of second kind integral equations. Wave Motion 5 (1983), $257-272$. 
[17] Rokhlin, V. Rapid solution of integral equations of scattering theory in two dimensions. Journal of Computational Physics 86 (1990), 414-439.

[18] Schwab, C., And Wendland, W. L. Kernel properties and representation of boundary integral operators. Mathematische Nachrichten 156 (1992), 187-218.

[19] Schwab, C., And Wendland, W. L. On numerical cubatures of singular surface integrals in boundary element methods. Numerische Mathematik 62 (1992), 343-369.

[20] Stein, E. Harmonic Analysis. Princeton University Press, Princeton, New Jersey, 1992.

[21] Taskinen, M., and Yla-Oijala, P. Current and charge integral equation formulation. IEEE Transactions on Antennas and Propagation 54 (2006), 58-67.

[22] Ying, L., Biros, G., AND Zorin, D. A high-order 3D boundary integral equation solver for elliptic PDEs in smooth domains. Journal of Computational Physics 219 (2006), 247-275.

[23] Young, P., Hao, S., and Martinsson, P.-G. A high-order nystrm discretization scheme for boundary integral equations defined on rotationally symmetric surfaces. Journal of Computational Physics 231 (2012), 4142-4159. 